\title{
Instructional strategies used in direct AAC interventions with children to support graphic symbol learning: A systematic review
}

(C) The Author(s) 2018 Reprints and permissions: sagepub.co.uk/journalsPermissions.nav DOI: I0.II 77/02656590I8755524 journals.sagepub.com/home/clt

(SAGE

\section{Yvonne Lynch}

Manchester Metropolitan University, UK

\section{Muireann McCleary \\ Central Remedial Clinic, Ireland}

\section{Martine Smith}

Trinity College Dublin, Ireland

\begin{abstract}
Augmentative and alternative communication (AAC) refers to a wide range of aided and unaided modes that are employed with a diverse group of people to support a range of language and communication outcomes. Children whose comprehension of spoken language greatly exceeds their ability to express themselves within that modality can be described as expressive users of AAC.

Interventions are important in promoting language acquisition and the expressive use of graphic symbols. Instructional strategies employed within interventions have an important impact on treatment effectiveness. A systematic review was undertaken to identify instructional strategies that have demonstrated effectiveness in supporting graphic symbol learning and aided language development in direct interventions with children aged 0-18 years who are expressive users of aided AAC (including children without learning difficulties and those with mild-moderate learning difficulties). A comprehensive search strategy was carried out and all studies meeting the inclusion criteria were quality appraised. A data extraction procedure was conducted on the studies meeting the quality appraisal criteria. Fifteen studies were included in the review investigating four instructional strategies used to support graphic symbol learning. The most studied instructional strategy, aided modeling, can be considered an evidenced-based practice. There is also strong research evidence to support the use of both narrative-based interventions and mand-model procedures to facilitate graphic symbol learning and aided language acquisition in children who
\end{abstract}


are expressive users of aided AAC. However, across the literature reviewed, a lack of consistent terminology hampered the ability to compare studies and draw conclusions. More consistent use of terminology would enhance the utility of the evidence base.

\section{Keywords}

aided language acquisition, augmentative and alternative communication, children, instructional strategies, intervention

\section{Introduction}

Augmentative and alternative communication (AAC) can be used to support language and communication in many different ways, using unaided and aided modalities. Unaided communication is expressed through resources internal to the communicator, such as gesture, eye gaze or facial expression. Aided communication involves the recruitment of external resources, such as pictures, graphic symbols or written words, displayed on low-tech communication books or boards, or using hightech options including speech generating devices (SGDs) and tablet technology. For some individuals, AAC modalities provide essential supports for both language comprehension and expression, a group that von Tetzchner and Martinsen (2000) categorized as alternative users of AAC. This group includes individuals such as those with multiple disabilities who may rely on visual supports both to understand their world and to express themselves within that world. However, AAC may also be introduced to support natural abilities, to augment unintelligible speech in specific situations or at specific points in development. For this group, the expectation is that natural speech may ultimately become a primary mode of communication. Children with learning disabilities or those with a diagnosis of childhood apraxia of speech may belong to this group. Finally, for some children and adults (expressive users in the von Tetzchner and Martinsen classification system), AAC modes provide a primary means of expression, usually to compensate for motor speech impairments (e.g. secondary to cerebral palsy). For this group, spoken language comprehension is relatively intact. The underlying presumption is that children require an expressive means to bypass their motor speech difficulties, but that over the course of development, they construct an internal speech-based language system as a basis for their expressive communication.

In many respects, the path to language and communication development for children who are expressive users of aided communication diverges from that of children who are developing typically. Aided communication development may be characterized by planned rather than spontaneous interactions (Light, 1997; von Tetzchner and Stadskleiv, 2016); communication interactions may be dominated by speaking partners in terms of distribution of the conversational floor (Raghavendra et al., 2012), and children must adapt to an asymmetry in input and output modalities of communication, with spoken language as their primary input mode, but an expectation that graphic symbols will function as the main output mode (Smith, 2006). As a result, language and communication development through augmented means does not occur naturally; rather, it requires specific intervention supports (Therrien et al., 2016).

The aim of any communication intervention is to instigate change, to prevent an undesirable outcome or to positively change the current position (Bunning, 2004). AAC interventions with young children aim to influence the underlying language development in children who use AAC forms (Thistle and Wilkinson, 2015). Intervention may involve a range of activities including direct interventions working with the child who uses AAC or indirect interventions working within the environment to effect change (Granlund et al., 2008). The focus of intervention may vary from targeting generic skills such as switch access, that may be used across multiple activities, (e.g. 
accessing an SGD as well as playing a computer game), to targeting AAC-specific skills such as using graphic symbols to communicate (Granlund et al., 2008). As such, AAC interventions are complex and comprise a range of interacting components. It is important to acknowledge that these different elements not only play a part in intervention outcomes in their own right, but may also have an interactive and integrative effect (Sevcik et al., 2009).

While ascertaining the effectiveness of complex interventions can be challenging (Campbell et al., 2007), not least because the contribution of multiple different components may be difficult to disentangle, it is important that clinicians use the available evidence base to inform intervention decisions. The instructional strategies (Beukelman and Mirenda, 2013) or procedures (Fey, 2006) used within interventions to lead to intervention goals are a key element of interventions. Fey (2006) describes intervention procedures (e.g. modeling the target, provision of structured practice, etc.) as the 'active ingredients of the intervention'. Given the resource demands of AAC interventions, it is imperative that the instructional strategies employed are both effective and efficient. While evaluating individual components of interventions in isolation may reduce the external validity of effectiveness research, it may provide useful indicators in selecting the most appropriate strategies to use in clinical practice. The aim of this systematic review is to identify instructional strategies that have demonstrated effectiveness in supporting graphic symbol learning and aided language development in direct interventions with children who are expressive users of AAC.

\section{Research question}

What instructional strategies are effective in supporting graphic symbol learning and aided language development for children who are expressive users of AAC?

\section{Method}

\section{Search procedure}

A multi-faceted search strategy was designed to identify relevant literature. Searches were conducted across four databases: Psychinfo (behavioural and social sciences), ERIC (education), CINAHL (nursing and allied health) and Pubmed (biomedical) to reflect the interdisciplinary nature of the AAC field (Schlosser et al., 2005). The database searches were supplemented by hand searches of the journal Augmentative and Alternative Communication and the Journal of Speech, Language and Hearing Research and citation searching. The search terms used were:

- 'Augmentative and Alternative Communication' AND 'Intervention’

- 'Aided Language Stimulation’ AND 'Intervention'

- 'Aided Language' AND 'Intervention'

- 'Augmented Language Intervention

- 'Graphic Symbols’ AND ‘Intervention'

\section{Inclusion criteria}

a Publication date and language. Studies written in the English language and published between 1992 and 2016 were included in the review. The initial searches were conducted on 30.12.2012. Given the rapid developments in technology over the previous two decades, a 20-year period was selected to capture interventions involving aided communication across this era of technological innovation. The searches were repeated and updated on 10.12.2016. 
b Participants. Study participants had to meet the criteria of (1) having a receptive-expressive language gap (with comprehension exceeding expression to comply with the categorization of expressive user of AAC), and (2) a developmental disability, (3) be aged 0-18 years and (4) with no identified social communication impairment. Studies for which participants did not meet the criteria for expressive user of AAC were excluded. Studies involving participants with a primary diagnosis of autism spectrum disorder (ASD) were excluded given that a social communication impairment forms part of the diagnostic criteria (American Psychiatric Association, 2013), and children with severe to profound intellectual disabilities were excluded as they are likely to use AAC to support both expression and comprehension. Studies with both eligible and ineligible participant data were only included if the results could be disaggregated.

c Intervention. Interventions within the area of AAC focused on graphic symbol learning and aided language acquisition were evaluated. All studies using direct intervention methods (i.e. that involved direct intervention with a target child) with the aim of developing graphic symbol learning or aided language acquisition were included. Indirect interventions such as communication partner training were excluded. Studies of challenging behaviour interventions, requesting/rejecting interventions and perceptions of interventions were excluded, as these studies did not address the process of graphic symbol learning. Similarly, studies that investigated AAC as a speech development technique were excluded. Finally, studies on the Picture Exchange Communication System (PECS) were also excluded. Bondy and Frost (1994) describe PECS as a programme to teach children with ASD a functional communication system. Given that PECS is primarily used with a population that had been excluded from the review and as it is a multifaceted approach that utilizes a specific communication context, these studies were excluded.

d Outcomes. Studies reporting outcome data on graphic symbol learning (receptive or expressive), symbol recall, the expressive use of graphic symbols (through the use of AAC), or outcome data on language acquisition in children using graphic-symbol-based AAC systems were included. Studies reporting outcomes related to specific operational competencies (e.g. how to use a scan pattern) were excluded. Studies that only reported outcomes related to literacy attainment were also excluded. However, studies with outcomes related to language and literacy were included, although only the data related to language achievements were evaluated. Papers that did not have outcome measures related to graphic symbol learning or aided language acquisition were excluded. Figure 1 details the search results across each stage of the systematic review.

\section{Screening process}

The search process yielded 1,756 records that were imported into Endnote for screening. The first author conducted a title and abstract review followed by full text review. Exclusion reasons were coded in a Participant, Intervention and Outcome format. Inter-rater reliability was conducted at the full text review stage. Two independent raters were provided with guidance and asked to review a sample of ten papers each (five of which had been included and five of which had been excluded by the first author). 100\% agreement was attained across all studies screened.

\section{Quality appraisal}

The inclusion/exclusion criteria were used to screen all returned records and sixty-six studies were identified for full text review. Full text review identified 24 studies for quality appraisal. Quality 




Figure I. Search results flowchart.

indicators were derived from Reichow et al.'s (2008) method. This evaluation tool can be used to evaluate methodological rigour and categorizes studies as strong, adequate or weak based on primary and secondary quality indicators (including specification of dependent variable, independent variable, visual analysis, fidelity and social validity). Fifteen studies attained ratings of adequate or strong and were included in the review. Nine of the 24 studies in the quality appraisal were categorized as weak according to Reichow et al.'s criteria (i.e. they attained fewer than four high-quality ratings on primary quality indicators or showed evidence of less than two secondary quality indicators). Studies attaining a weak rating were removed from the review (see supplemental material for full details of quality appraisal ratings).

The included studies were also appraised collectively. Horner et al. (2005) propose that in order to be considered evidence-based, a practice must have a minimum of five single-subject studies that meet acceptable level of methodological rigour and quality criteria and that are published in peer-review journals. In addition, studies must be conducted by at least three different researchers across three or more geographical locations and must collectively include a minimum of 20 participants (Horner et al., 2005). This standard was applied to the studies in the present review to ascertain if the instructional strategies investigated can be considered evidence-based practices. 


\section{Inter-rater agreement}

Seven AAC clinicians applied the criteria to the 24 studies considered for the review. Each clinician independently carried out quality appraisal of at least two studies. Discrepancies between raters arose across four studies. These studies were appraised for a third time by an independent rater. Where there was $100 \%$ agreement between two of three raters, their agreed rating was applied.

\section{Data extraction}

A data extraction template was developed based on the research question and used to extract the following study characteristics: sample size, age and diagnosis of participants, primary focus of intervention, instructional strategies used, dosage (i.e. the amount and frequency of intervention), outcome measures used and intervention outcomes. Table 1 sets out the data extracted from the 15 studies in the review. The studies were published from 1995-2015.

\section{Results}

Across the 15 included studies, four instructional strategies were identified, that met the criteria for provisional consideration as effective in supporting graphic symbol learning and/or aided language acquisition. These strategies are:

- Aided AAC modeling: the provision of augmented input alongside spoken language in naturalistic settings (Sennott et al., 2016)

- Narrative-based interventions: the provision of aided AAC modeling and language elicitation techniques embedded in a narrative routine.

- An eclectic approach: the provision of communication opportunities, and aided AAC modeling with least to most prompt hierarchies to facilitate symbol production.

- A mand-model instructional strategy: the provision of clinician-led communication opportunities with a hierarchy of prompts. Children were asked to produce graphic symbol output; if they did not respond to prompts, a model was provided.

Each instructional strategy studied incorporated a number of techniques to support aided language acquisition and graphic symbol learning. While there was some overlap across strategies, the manner in which they were used and the aim of the strategies varied. For example, aided language modeling primarily focused on re-balancing the input-output asymmetry experienced by children who use AAC and symbol output by the child was not directly targeted. Narrative interventions and eclectic approaches focused on both augmented input and on elicitation of symbol output. Finally, in the mand-model strategy, the focus was on symbol production/selection as a means to learn target linguistic structures. The included studies provide evidence of the effectiveness of these four instructional strategies in supporting learning across a number of domains as detailed below.

\section{Aided language modeling (studies I, 2, 3, 5, 6, 8, 10 and I4)}

Over half the included studies examined aided modeling strategies. The included studies suggest that the use of augmented input is supportive of symbol comprehension (Dada et al., 2009; Harris et al., 2004), expressive symbol production (Harris et al., 2004; Iacono and Duncum, 1995; 


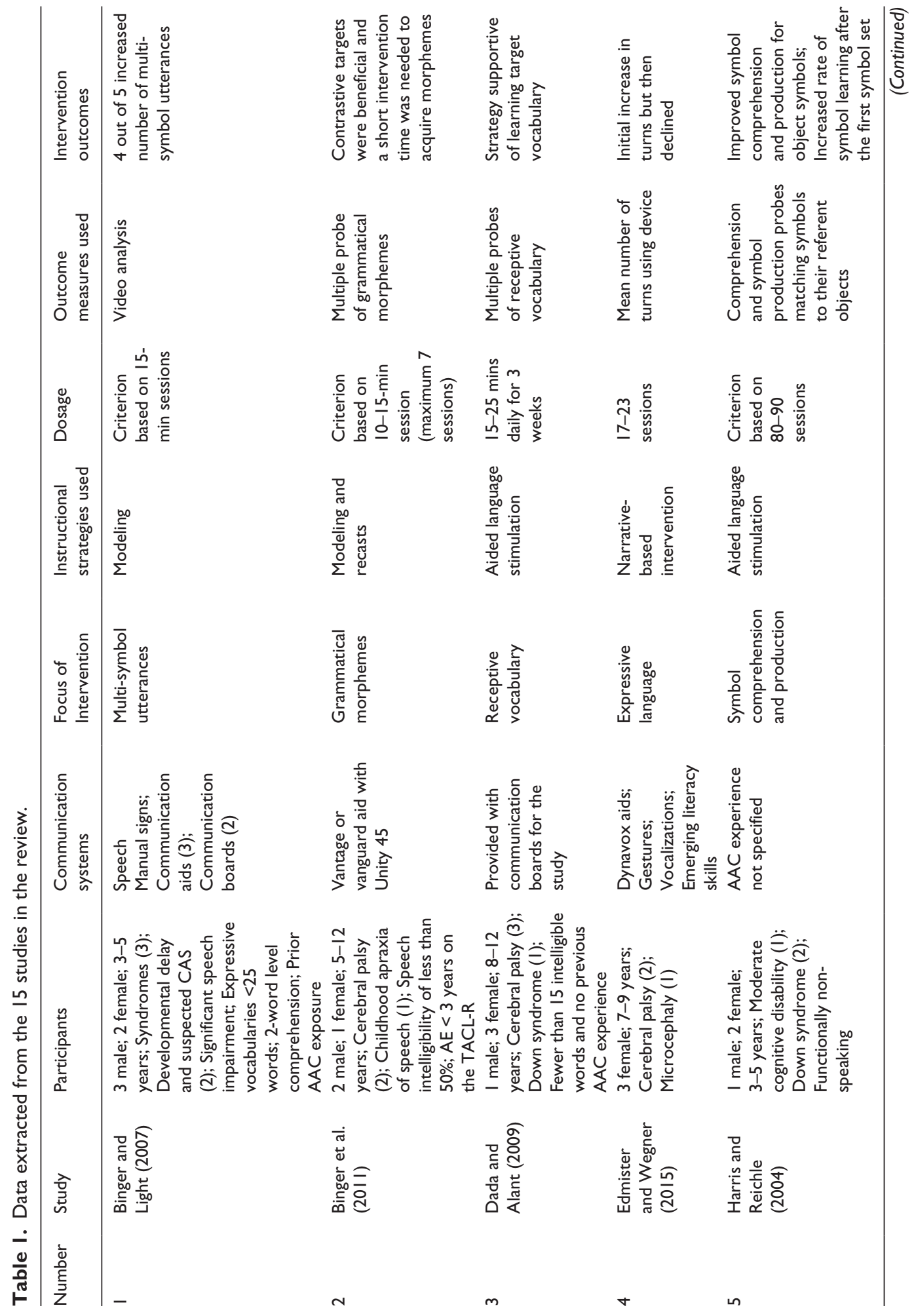




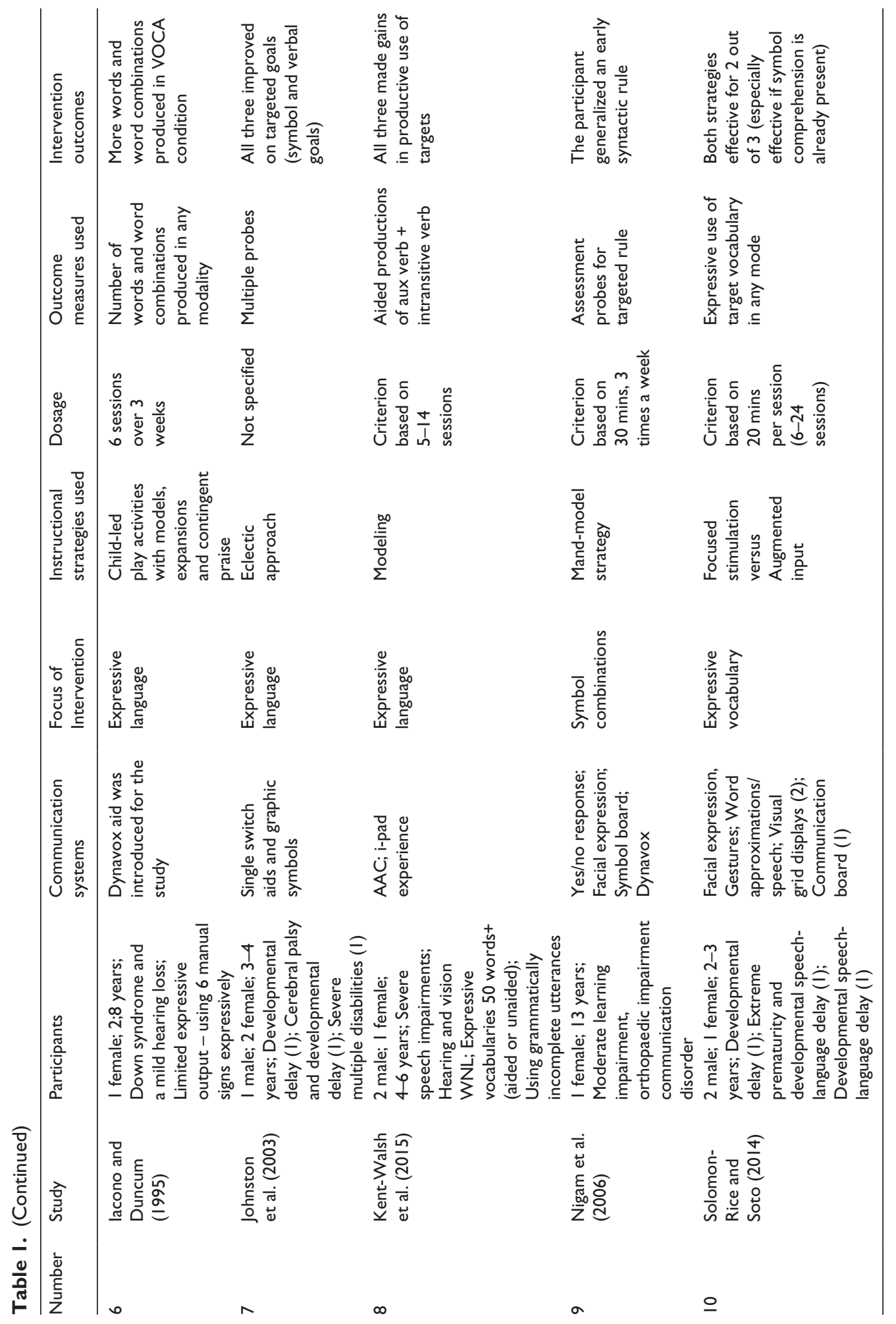







Solomon-Rice et al., 2014; Taylor et al., 2003) and the use of multi-symbol utterances and word combinations (Binger et al., 2011; Iacono and Duncum, 1995). The studies also suggest aided modeling may facilitate acquisition of language structures such as grammatical morphemes (Binger et al., 2007) and auxiliary verb and intransitive verb combinations (Kent-Walsh et al., 2015). The studies on aided modeling were also considered collectively using Horner et al.'s (2005) criteria for determining if a practice can be considered evidence based. Aided language modeling met all the criteria to be considered evidence-based and it was the only strategy in the review to meet all the required criteria.

\section{Narrative-based intervention (studies 4, II, I2 and I3)}

Four studies examined narrative-based interventions. Three studies reported increased linguistic complexity in the aided output of participants post-intervention. Participants demonstrated increases in the number and diversity of symbols produced and an increased use of multi-symbol utterances (Soto et al., 2008, 2009). Two studies also reported an improvement in narrative complexity (with outcomes of improved plot structures and increased cohesion and coherence) (Soto et al., 2008, 2009). The fourth study explored the effect of repeated storybook reading on the number of communicative turns (Edmister et al., 2015). Although two out of three participants initially demonstrated an increase in their use of symbol-based communicative turns, these gains were not maintained across the intervention.

Narrative-based instructional strategies may be effective in supporting expressive language development; however, caution is needed in generalizing from these findings due to the small participant numbers across the included studies for this instructional strategy and the variable profile of gains across participants.

\section{Eclectic approach (study 7)}

Johnston et al. (2003) applied an eclectic approach (i.e. increased communication opportunities, aided modeling, hierarchy of prompting) that they reported supported three participants in achieving targeted goals and in increasing expressive communication (both verbal and symbol-based).

\section{Mand-model procedure with matrix strategy (studies 9 and I5)}

Two studies applied a mand-model procedure. One explored the use of a mand-model procedure to support the acquisition of an Action+Object rule using graphic symbols (Nigam et al., 2006). One out of three participants met the inclusion criteria of the current review. That participant learned the Action+Object rule and was able to generalize it to combinations of graphic symbols not targeted in the intervention. In the second study (Tönsing et al., 2014), three out of four participants increased production of multi-symbol combinations targeted and generalized to non-trained exemplars. The remaining participant did not reach criterion in the maximum number of sessions, a profile the authors suggest may have been related to distractibility and disengagement with the intervention activity. These two studies suggest that a mand-model strategy may be supportive of expressive aided syntax development.

\section{Discussion}

At the heart of AAC interventions for children must be a focus on supporting linguistic development and expressive communication through aided means. The present review suggests the 
evidence base for instructional strategies to promote language development is emerging. However, further research is warranted to enable clinicians to choose optimal instructional strategies.

Considering the studies collectively, one strategy, aided modeling, met Horner et al.'s (2005) criteria as an evidence-based practice. Given that one of the challenges faced by children acquiring language using aided communication is that they receive input primarily through speech but must express themselves using graphic symbols, the effectiveness of aided modeling as a strategy may derive from the fact that it offers an opportunity to observe competent language users using symbols for communication and to receive symbols as input. Indirect benefits may derive from the fact that aided modeling may require communication partners to slow their rate of speech and may highlight for them the challenges of using aided communication leading to other positive communication behaviours (Smith, 2015; von Tetzchner and Stadskleiv, 2016). To have one instructional strategy meet criteria for evidence-based effectiveness is a step forward and lends support to clinicians in advocating for augmented input across communication settings. While the remaining three strategies did not meet the criteria set down by Horner et al, they nonetheless seem promising. As these interventions incorporated use of aided modeling as one instructional strategy, it is not possible to determine whether the additional components (increased communication opportunities and language elicitation techniques) represent 'added value' as independent instructional strategies, or whether the benefit of this intervention approach is related to the provision of a context into which aided language modeling can be readily imported. Similarly, both the eclectic approach and the studies involving use of mand-model procedures incorporated some use of aided modeling, although in the case of mandmodel procedures the focus was on specific linguistic targets within structured teaching contexts.

Unlike the studies focused on aided language modeling, the narrative-based, eclectic and mandmodel studies all incorporated a focus on symbol production as well as aided modeling. Production opportunities may represent important contexts for learning that complement what is available through aided modeling (Smith, 2015). What is not clear from the available evidence base is whether the benefits of these strategies apply equally at all stages of aided language development, or whether there may be differential benefit from selected use of a specific strategy at key points in development (Nelson, 1992).

Although the current review provides some support for use of four instructional strategies to support graphic symbol learning and aided language development, it does not address the question of relative effectiveness and efficiency. As no comparative studies were identified in the review, it was not possible to compare any of the identified strategies in terms of effectiveness and efficiency. The majority of the studies in the review focused on naturalistic strategies which may take advantage of naturally occurring communicative opportunities. A criticism of naturalistic strategies is they may not provide the range and quantity of linguistic opportunities as more structured clinician-led strategies. While the evidence base is emerging, it warrants further development to enable clinicians to make informed decisions for their clients.

\section{Limitations}

The present review has a number of limitations that must be acknowledged. First, some of the search criteria decisions introduced biases, namely a language bias and a publication bias (only English language studies published in peer-reviewed journals were included). Second, the review focused on children who are expressive users of AAC. This group was chosen to allow a concentrated consideration of graphic symbol learning in children who are primarily using aided AAC as an expressive mode. Therefore, the findings are not applicable to other groups of children and adults who use AAC (for example, those who use AAC to support comprehension and expression) or to other outcomes (for example, the impact of AAC on speech development). Third, the studies 
provided limited information on the stages of aided development in the child participants studied. The review suggests that naturalistic strategies are supportive of graphic symbol learning. However, it is not possible to comment on whether naturalistic strategies are particularly effective at different stages of aided language development or if other strategies may be more effective for particular aspects of aided language learning. Further research is warranted to compare the effectiveness of instructional strategies at different stages of aided language acquisition. Finally, the variable use of terminology across the AAC literature presented challenges in identifying studies for inclusion in the review. As a result, a number of additional searches were conducted to ensure search robustness. Furthermore, the variability in terminology used across the literature presented difficulties in evaluating the studies. Different terms were used to describe the same or similar strategies (for example, modeling and aided language stimulation) (see also Sennott et al., 2016). Due to the lack of consistent use of terminology, drawing conclusions across studies was challenging. For example, the study by Dada and Alant (2009) was the only one to define aided modeling (aided models were presented with spoken language input at least 70\% of the time and a ratio of 80:20 of statements to questions). Even though many of the studies described the intervention undertaken and addressed treatment fidelity, there were assumptions in many studies that the strategies outlined required no operational definition. The variability in the use of terminology and the difficulty ascertaining how exactly terms should be interpreted across studies presents a real challenge to the field in terms of building an evidence base.

\section{Conclusions}

Interventions to support the needs of children who rely on aided communication are complex and multi-faceted. Based on a small but emerging evidence base, this review suggests that at least four intervention strategies are potentially effective in supporting graphic symbol learning and aided language development. These strategies essentially rest on provision of accessible input in meaningful linguistic contexts, mirroring the findings from research with children with language impairment (Fey, 1986). However, in order to be fully effective, such strategies must be embedded in interventions that reflect the complexity of interactions involving aided communication and focused on enhancing participation rather than addressing development of isolated skills.

\section{Acknowledgements}

Thank you to the SLT team at the Central Remedial Clinic for their support with the quality appraisal process and Jennifer O'Brien for her help conducting some of the searches.

\section{Declaration of conflicting interests}

The author(s) declared no potential conflicts of interest with respect to the research, authorship, and/or publication of this article.

\section{Funding}

The author(s) disclosed receipt of the following financial support for the research, authorship, and/or publication of this article: The first author received a grant from the Central Remedial Clinic's Research Trust which part funded her doctoral studies that included this review.

\section{ORCID iD}

Yvonne Lynch (iD https://orcid.org/0000-0003-3209-3099 


\section{References}

Note. Studies included in the review are denoted with an asterisk.

American Psychiatric Association (2013) Diagnostic and statistical manual of mental disorders. 5th edition. Washington, DC: American Psychiatric Association.

Beukelman D and Mirenda P (2004) Augmentative and alternative communication: Management of severe communication disorders in children and adults. Baltimore, MD: Brookes.

*Binger C and Light J (2007) The effect of aided AAC modeling on the expression of multi-symbol messages by preschoolers who use AAC. Augmentative and alternative communication 23: 30-43.

*Binger C, Maguire-Marshall M, and Kent-Walsh J (2011) Using aided AAC models, recasts and contrastive targets to teach grammatical morphemes to children who use AAC. Journal of Speech, Language and Hearing Research 54: 160-176

Bondy A and Frost L (1994) The picture exchange communication system. Focus on autism and other developmental disabilities 9: 1-19

Bunning K (2004) Speech and language therapy intervention: Frameworks and processes. London, Whurr.

Campbell NC, Murray E, Darbyshire J, et al. (2007) Designing and evaluating complex interventions to improve healthcare. British Medical Journal 2007(7591): 334-445.

*Dada S and Alant E (2009) The effect of aided language stimulation on vocabulary acquisition in children with little or no functional speech. American Journal of Speech-Language Pathology, 18: 50-64.

*Edmister E and Wegner J (2015) Repeated reading, turn taking, and augmentative and alternative communication (AAC). International Journal of Disability, Development, and Education 62: 319-38.

Fey ME (1986) Language intervention with young children. San Diego, CA: Taylor and Francis.

Fey ME (2006) Introduction. In: McCauley RJ and Fey ME (eds) Treating of language disorders in children (pp. 1-20). London: Paul H Brookes.

Granlund M, Bjorck-Akesson E, Wilder J, and Ylven R (2008) AAC interventions for children in a family environment: Implementing evidence in practice. Augmentative and Alternative Communication 24: 207-19.

*Harris MD and Reichle J (2004) The impact of aided language stimulation on symbol comprehension and production in children with moderate cognitive disabilities. American Journal of Speech-Language Pathology 13: 155-67.

Horner RH, Carr E, Halle J, McGee G, Odom S, and Wolery M (2005) The use of single-subject research to identify evidence-based practice in special education. Exceptional Children 71: 165-79.

*Iacono T and Duncum J (1995) Comparison of sign alone and in combination with an electronic communication device in early language intervention: case study. Augmentative and Alternative Communication 11: $249-59$.

*Johnston SS, McDonnell AP, Nelson C, and Magnavito A (2003) Teaching functional communication skills using augmentative and alternative communication in inclusive settings. Journal of Early Intervention 25: $263-80$.

*Kent-Walsh J, Binger C, and Buchanan C (2015) Teaching children who use augmentative and alternative communication to ask inverted yes/no questions using aided modeling. American Journal of SpeechLanguage Pathology 24: 222-36.

Light J (1997) 'Let's go star fishing': Reflections on the contexts of language learning for children who use aided AAC. Augmentative and Alternative Communication 13: 158-71.

Nelson NW (1992) Performance is the prize: Language competence and performance among AAC users. Augmentative and Alternative Communication 8: 3-18.

*Nigam R, Schlosser R, and Lloyd LL (2006) Concomitant use of the matrix strategy and the mand-model procedure in teaching graphic symbol combinations. Augmentative and Alternative Communication 22: $160-77$.

Raghavendra P, Olsson C, Sampson J, McInerney R, and Connell T (2012) School participation and social networks of children with complex communication needs, physical disabilities, and typically developing peers. Augmentative and alternative communication 28: 33-43.

Reichow B, Volkmar F, and Cicchetti DV (2008) Development of an evaluative method for evaluating and determining evidence-based practices in autism. Journal of Autism and Developmental Disabilities 38: 1311-9. 
Schlosser R, Wendt O, Angermeier KL, and Shetty M (2005) Searching for evidence in augmentative and alternative communication: Navigating a scattered literature. Augmentative and Alternative Communication 21: $233-55$.

Sennott SC, Light J, and McNaughton D (2016) AAC modeling intervention research review. Research and Practice for Persons with Severe Disabilities 41: 101-15.

Sevcik RA, Romski MA, and Adamson LB (2009) Measuring AAC interventions for individuals with severe disabilities. Augmentative and Alternative Communication 15: 38-44.

Smith M (2006) Speech, language and aided communication: Connections and questions in a developmental context. Disability and Rehabilitation 28: 151-7.

Smith MM (2015) Language development of individuals who require aided communication: Reflections on state of the science and future directions. Augmentative and Alternative Communication 31:215-23.

*Solomon-Rice P and Soto G (2014) Facilitating vocabulary in toddlers using AAC: A preliminary study comparing focused stimulation and augmented input. Communication Disorders Quarterly 35: 204-15.

*Soto G and Dukhovny E (2008) The effect of shared book reading on the acquisition of expressive vocabulary of a 7 year old who uses AAC. Seminars in Speech and Language 29: 133-45.

*Soto G, Solomon-Rice P, and Caputo M (2009) Enhancing the personal narrative skills of elementary school-aged students who use AAC: The effectiveness of personal narrative intervention. Journal of Communication Disorders 42: 43-57.

*Soto G, Yu B, and Kelso J (2008) Effectiveness of multifaceted narrative intervention on the stories told by a 12-year-old girl who uses AAC. Augmentative and alternative communication 24: 76-87.

*Taylor R and Iacono T (2003) AAC and scripting activities to facilitate communication and play. Advances in Speech Language Pathology 5: 79-93.

Therrien MC, Light J, and Pope L (2016) Systematic Review of the effects of interventions to promote peer interactions for children who use Aided AAC. AAC: Augmentative and Alternative Communication 32: 81-93.

Thistle JJ and Wilkinson KM (2015) Building evidence-based practice in AAC display design for young children: Current practices and future directions. Augmentative and Alternative Communication 31: 124-36.

*Tönsing KM, Dada S, and Alant E (2014) Teaching graphic symbol combinations to children with limited speech during shared story reading. Augmentative and Alternative Communication 30: 279-97.

von Tetzchner S and Martinsen H (2000) Introduction to augmentative and alternative communication. $2 \mathrm{nd}$ edition. London: Whurr.

von Tetzchner S and Stadskleiv K (2016) Constructing a language in alternative forms In: Smith MM and Murray J (eds) The silent partner? Language, interaction and aided communication. J\&R Press: Croydon 